\title{
A Case of COVID-19 Failed in Viral Identification but Showed Consistent Host Responses to Severe Acute Respiratory Syndrome Coronavirus 2
}

\author{
Yoshitaka Maeda ${ }^{\mathrm{a}, \mathrm{c}}$, Hiroyuki Miyo ${ }^{\mathrm{b}}$, Atsuki Ohashia, ${ }^{\mathrm{a}}$, \\ Takanobu Yamamoto ${ }^{\mathrm{b}}$, Tomoyuki Ogata ${ }^{\mathrm{b}}$
}

\begin{abstract}
In a 75-year-old hemodialysis patient, pneumonia manifested with computed tomography (CT) images suggestive of coronavirus disease 2019 (COVID-19). Severe acute respiratory syndrome coronavirus 2 (SARS-CoV-2) ribonucleic acid (RNA) was not detected with repeated polymerase chain reaction (PCR) tests, but serum immunoglobulin $\mathrm{G}(\mathrm{IgG})$ and IgM antibodies against SARS-CoV-2 turned positive with two different assay kits. The patient was thus treated as a probable COVID-19 case, and then recovered through the intensive care including ciclesonide, favipiravir, and methyl prednisolone. In PCRnegative cases, the diagnosis of COVID-19 cannot be established at present, but such COVID-19 cases certainly exist. To avoid a delay in treatment of COVID-19, available diagnostic tools should be utilized with careful attention for their limitations.
\end{abstract}

Keywords: SARS-CoV-2; Ciclesonide; Favipiravir; ESKD

\section{Introduction}

Severe acute respiratory syndrome coronavirus 2 (SARSCoV-2) infection had become pandemic in the world [1], and more than twenty million people have cumulatively suffered from coronavirus disease 2019 (COVID-19). At present, the number of the patients was increasing by more than 200,000 every day. Japanese patients had been reported relatively less than those of other countries $(0.25 \%$ of total patients in the world), which might result from several hypothetical reasons

Manuscript submitted August 12, 2020, accepted August 19, 2020

Published online August 28, 2020

${ }^{a}$ Nephrology Division, Department of Internal Medicine, JA Toride Medical Center, Toride, Ibaraki 302-0022, Japan

'bnfection Control Team, Department of Internal Medicine, JA Toride Medical Center, Toride, Ibaraki 302-0022, Japan

${ }^{\mathrm{c} C}$ Corresponding Author: Yoshitaka Maeda, Nephrology Division, Department of Internal Medicine, JA Toride Medical Center, 2-1-1 Hongo, Toride, Ibaraki 302-0022, Japan. Email: yoshimaeda43@yahoo.co.jp

doi: https://doi.org/10.14740/jmc3566
[2]. Otherwise, the diagnostic system in Japan might be simply insufficient for full screening of suspected cases [3].

Generally, SARS-CoV-2 is identified by polymerase chain reaction (PCR) method of viral ribonucleic acid (RNA) from nasal swab samplings. However, the sensitivity of this method is not satisfactory, thus repeated measurements were recommended for PCR-negative cases to reach a final diagnosis [4]. We experienced a case with negative PCR results for five times, in whom positive antibodies against SARS-CoV-2 were found with two different assay kits. The PCR method was recognized as an essential tool in establishing a diagnosis of COVID-19 [1]. However, this method alone may cause a falsenegative diagnosis, underestimate the real prevalence rate of COVID-19, and delay treatment against COVID-19. To deal with such PCR-negative cases with a suspicion of COVID-19 appropriately, we thought it was important to report the case whom we experienced recently.

\section{Case Report}

A 75-year-old male visited JA Toride Medical Center with complaints of fever $\left(37.6^{\circ} \mathrm{C}\right)$ and general fatigue before a hemodialysis session. Maintenance hemodialysis was undertaken thrice a week for the past 2 years at the same hospital. The patient was a permanent non-smoker, but he had a long history of poorly controlled type 2 diabetes mellitus resulting in endstage kidney failure (ESKD). According to the blood examination 1 week before this episode, hemoglobin A1c (HbA1c) was $8.7 \%$ and glycoalbumin was $35.6 \%$. Moreover, a pacemaker had been implanted for treatment of complete atrioventricular block since 2006. He did not get vaccinated for influenza in the last year.

On visit, he had no apparent respiratory symptoms with normal breath sound, and there were no remarkable physical findings except for mild leg edema. However, images of plain chest X-ray (Fig. 1) and chest computed tomography (CT) (Fig. 2) revealed a consolidative shadow in the S6 region of the right lung, which suggested bacterial pneumonia, rather than pneumonia due to COVID-19. As Tables 1 and 2 show, the blood examination showed normal count and fraction of leukocytes and slight elevation in C-reactive protein (CRP). Because the patient had oliguric, urinary antigens of Streptococ- 


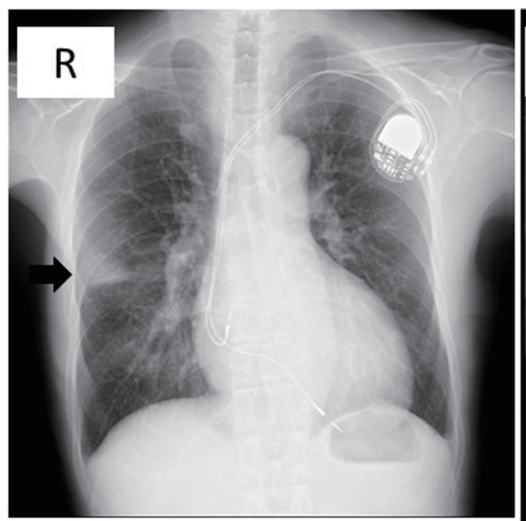

Day 1

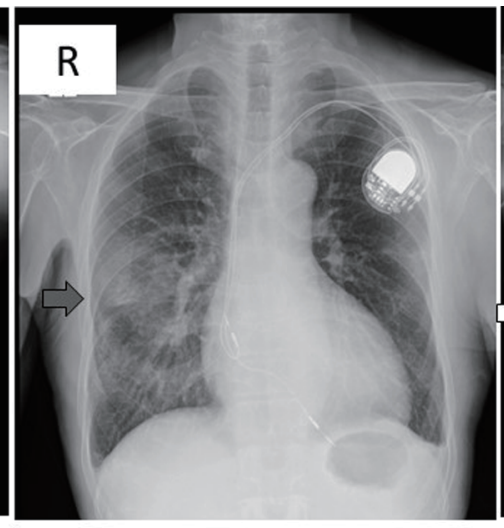

Day 8

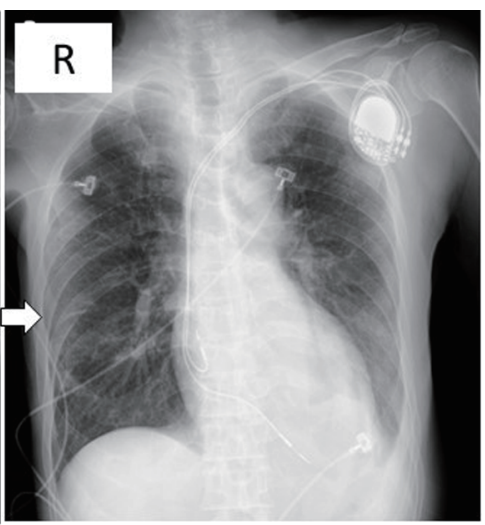

Day 28

Figure 1. Chest X-ray images on day 1, 8, and 28. A wedge-shaped opacity with a relatively clear margin was observed in the right middle lung zone (black arrow) on day 1 . That opacity turned blurred and extended in the lower lung field (gray arrow) on day 8 , then diminished (white arrow) on day 28. The radiolucency of left lower lung field was reduced on day 28 due to pleural effusion (see Fig. 2).

cus and Legionella species were not be able to be tested. Since the hospital accepted COVID-19 patients at that time, a nasal swab sampling was carried out for a reverse transcription-PCR (RT-PCR) test for excluding SARS-CoV-2 infection. The RTPCR was conducted through the established protocol [5] at Ibaraki Prefectural Institute of Public Health (Ibaraki, Japan).

Then he had been followed up at the outpatient with oral administration of $400 \mathrm{mg}$ of moxifloxacin (MFLX) daily until day 10. But his condition had not been improved with this treatment alone. To rule out COVID-19, additional RT-PCR tests were done at the institute described above on days 5, 8, 18, and at another laboratory, SRL Inc. (Tokyo, Japan) on day 10 (Table 2). But their results were all negative. Simultaneous blood culture study also revealed no growth of infectious microorganisms.

On day 10, since the initial consolidation became blurred, and appeared like ground glass opacification (GGO), and similar shadows were extensively found in the contralateral lung in the chest CT scans (Fig. 3), the patient was admitted to the hospital with a probable diagnosis of COVID-19. He had no contact history with other COVID-19 patients treated in this hospital. Meanwhile, he was a city council member, and usually worked with a variety of people. Thus, his actual exposure timing to SARS-CoV-2 was not been specified. His son, only a family member living together, showed a negative PCR result of SARS-CoV-2 RNA, although his serum antibodies against SARS-CoV-2 had not been examined.

After obtaining the informed consent from the patient according to the approval by the ethical committee of JA Toride Medical Center on the administration of ciclesonide and favipiravir against COVID-19 cases, inhalation therapy of $200 \mu \mathrm{g}$ of ciclesonide, twice a day was started on his admission, and $3.6 \mathrm{~g} /$ day of favipiravir was initially administered on day 12 , then $1.6 \mathrm{~g}$ /day was continued until day 25 (Fig. 4). To treat possible concomitant bacterial pneumonia, $500 \mathrm{mg}$ of meropenem (MEPM) had been simultaneously administered between day



Figure 2. Axial chest CT images on day 1, 10, and 29. An opacity found in the chest X-ray (Fig.1) was consolidative in S6 of the right lung (black arrow) on day 1. That consolidation became blurred and extended as GGO (thin gray arrow) and similar shadows also appeared in the left lung (thick gray arrow) on day 10. These shadows diminished except for left pleural effusion on day 29 (white arrow). That effusion was gradually decreasing with adjustment of body fluid by dialysis sessions. CT: computed tomography; GGO: ground glass opacification. 
Table 1. Laboratory Findings Before the Dialysis Session on Day 1

\begin{tabular}{|c|c|c|}
\hline Parameter & Value & Unit \\
\hline White blood cells & 4,630 & $/ \mu \mathrm{L}$ \\
\hline Neutrophils & 76.8 & $\%$ \\
\hline Lymphocytes & 11.2 & $\%$ \\
\hline Basophils & 0.4 & $\%$ \\
\hline Eosinophils & 4.3 & $\%$ \\
\hline Monocytes & 7.3 & $\%$ \\
\hline Red blood cells & 353 & $\times 10^{4} / \mu \mathrm{L}$ \\
\hline Hemoglobin & 11.4 & $\mathrm{~g} / \mathrm{dL}$ \\
\hline Hematocrit & 33.5 & $\%$ \\
\hline Platelets & 7.3 & $\times 10^{4} / \mu \mathrm{L}$ \\
\hline Total protein & 6.6 & $\mathrm{~g} / \mathrm{dL}$ \\
\hline Albumin & 3.8 & $\mathrm{~g} / \mathrm{dL}$ \\
\hline Blood urea nitrogen & 73 & $\mathrm{mg} / \mathrm{dL}$ \\
\hline Creatinine & 9.46 & $\mathrm{mg} / \mathrm{dL}$ \\
\hline $\mathrm{Na}$ (Sodium) & 136.3 & $\mathrm{mEq} / \mathrm{L}$ \\
\hline K (Potassium) & 4.61 & $\mathrm{mEq} / \mathrm{L}$ \\
\hline Chloride & 96.3 & $\mathrm{mEq} / \mathrm{L}$ \\
\hline Calcium & 8.4 & $\mathrm{mg} / \mathrm{dL}$ \\
\hline Inorganic phosphorus & 4.5 & $\mathrm{mg} / \mathrm{dL}$ \\
\hline AST & 32 & $\mathrm{U} / \mathrm{L}$ \\
\hline ALT & 27 & $\mathrm{U} / \mathrm{L}$ \\
\hline Glucose & 215 & $\mathrm{mg} / \mathrm{dL}$ \\
\hline
\end{tabular}

AST: aspartate aminotransferase (GOT); ALT: alanine aminotransferase (GPT)

12 and day 23. Saturation of percutaneous oxygen $\left(\mathrm{SpO}_{2}\right)$ of the patient had been kept above 95\% (95-100\%) with 1 - $2 \mathrm{~L} /$ min of oxygen inhalation through a nasal cannula (Fig.4).

As Table 2 indicates, immunoglobulin M (IgM) antibodies against SARS-CoV-2, initially negative on day 1, were detected from his serum sample with an assay kit (One Stop COVID19 IgM/IgG Antibody Test, Kyokuto Pharmaceutical Industrial Co., Tokyo, Japan) on day 10. IgG antibodies also turned positive on day 15. IgM antibodies against SARSCoV-2 were also ascertained positive with another assay kit (Immunochromatographic Test Kit, Kurabo Industries Co, LTD, Osaka, Japan) on day 21.

His inflammatory signs, such as his body temperatures and serum CRP levels had been transiently suppressed during days 22 and 25, but these signs had elevated again from day 26. MEPM, $0.5 \mathrm{~g} / \mathrm{day}$, was resumed, and $40 \mathrm{mg} /$ day of methyl prednisolone was added to prevent a possible cytokine storm caused by COVID-19, because pulmonary infiltrative shadows were rather improved (Figs. 1, 2), both of serum interleukin 6 (IL-6) and D-dimer levels elevated (Table 2), and the blood culture test showed no bacterial growth (Table 2). Antifungal agents were not required, although elevation in serum $\beta$-Dglucan level was noted in his recovery phase. With these treat- ments, his inflammatory signs were reduced, and his general condition recovered around 35 days after the onset (Fig. 4).

\section{Discussion}

We experienced a probable case of COVID-19 with positive antibodies against SARS-CoV-2 and typical images in chest CT scans, but negative RT-PCR for five times from day 1 to 18 after the onset of symptoms.

RT-PCR methods had showed unsatisfactory sensitivities for detecting SARS-CoV-2 RNA, especially with samples not directly through the lower respiratory tract [6]. There are several reasons for this limitation. Besides inadequate samplings, viral infiltration might be restricted to the lower respiratory tract in some cases, which may cause a failure to identify SARS$\mathrm{CoV}-2$ with samplings only from the upper respiratory tract, such as nasal swab samplings [6]. Moreover, a detection rate of viral RNA was dependent on a timing of sampling. A rate was the least on the day of exposure, then increasing, and the highest around 8 days after exposure (3 days after the onset) [7]. Finally, mutation of viral RNA, reported more frequently than expected [8], also causes a detection failure with conventional PCR procedures, in which primers for PCR should be adjusted for newly mutated genes. Similar concerns were also required for specificity of antibody assays for SARA-CoV-2, since viral antigenicity would change in accordance with viral genomics.

In an epidemiological study, Ai et al reported probable COVID-19 cases with negative RT-PCR results, although the patients showed positive infiltration shadows in the chest CT [9]. According to their report, such cases occupied 308 of 1,014 patients, and 49 of them showed repeated negative RT-PCR results.

In the case reported here, since the RT-PCR test was repeated for five times over 2 weeks, SARS-CoV-2 infection might be restricted in the lower respiratory tract. In future clinical settings, samplings directly from the lung or from stools will be possible alternates in similar cases, although the former increase a practitioner's exposure risk for SARS-CoV-2, and the latter requires purification steps of stool samples before proceeding to PCR tests.

In assay kits for detecting antibodies against SARSCoV-2, specificity of each antibody to SARS-CoV-2 has not been clarified yet $[10,11]$, and some assay kits might detect other coronavirus subspecies, or show pseudo-positive reaction due to artifacts, such as autoantibodies of patients. In this case, IgG and IgM antibodies against SARS-CoV-2 were initially negative, then IgM, and subsequently IgG turned positive. In addition, IgM antibodies were also detected using another assay system. Moreover, non-specific antibodies against human immunoglobulins such as rheumatoid factor were not found. Thus, it seemed unlikely that detected antibodies had been produced through immune responses to coronavirus subspecies other than SARS-CoV-2, or non-specific sensing of human immunoglobulins.

Chest CT images are recommended to be utilized as first screening tools for suspicious COVID-19 cases [12]. However, there is no specific finding for COVID-19. Lung shadows in COVID-19 are commonly found in some other viral infec- 


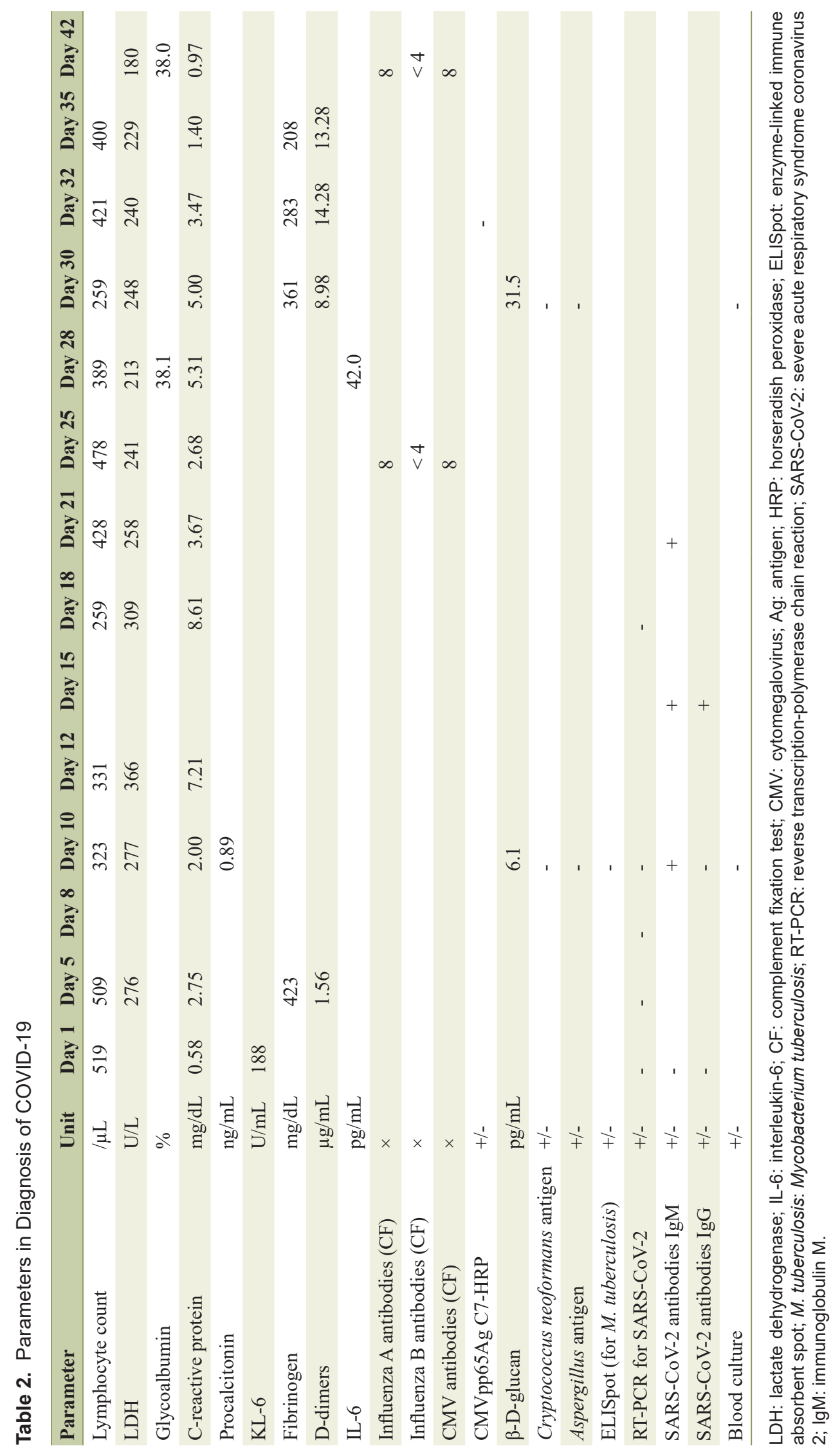




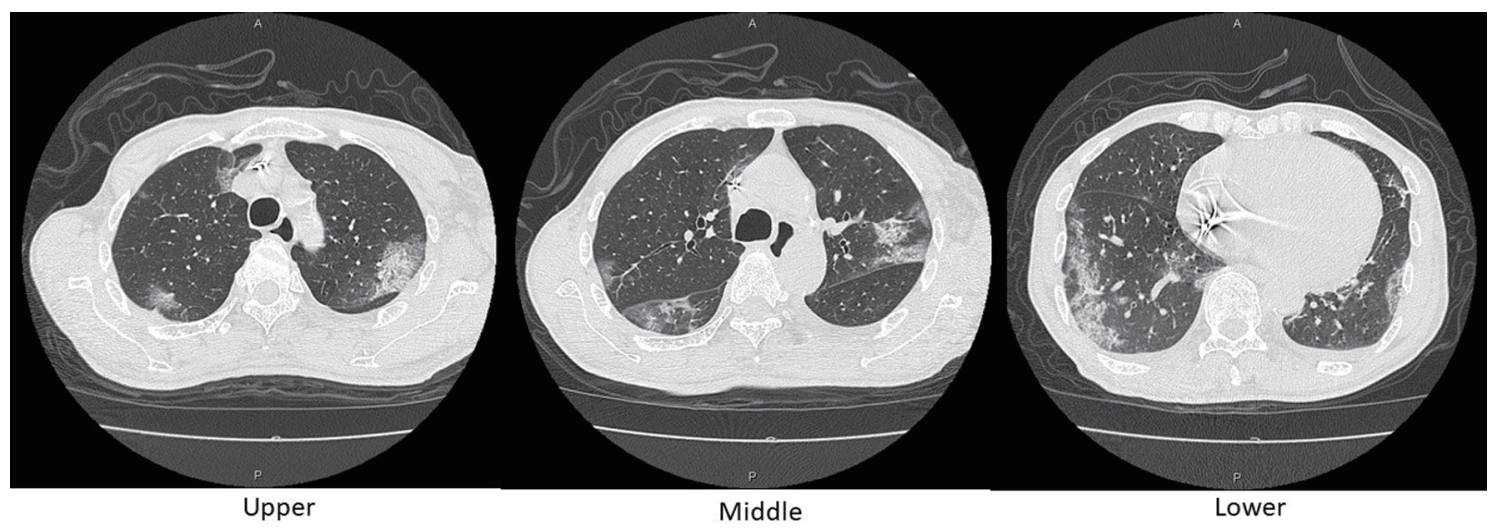

Figure 3. Axial chest CT images on day 10. Reticular shadows with a peripheral distribution, partially with crazy-paving appearance, scattered in the bilateral lungs. CT: computed tomography.

tions or interstitial pneumonitis. Meanwhile, shadows similar to bacterial pneumonia are often observed even in true COVID-19 cases. This case also showed consolidation suggesting bacterial pneumonia at the initial phase, but its shadow then changed its density and diversity. Moreover, other shadows appeared in the contralateral lung, and CT images turned to relatively typical patterns for COIVD-19 with bilateral, multifocal, and peripherally distributed shadows on day 10 (Figs. 2, 3). Similar transition patterns of pulmonary lesions were often reported [13]. Generally, consolidations were increasing with disease progression of COVID-19 [14]. This case showed rather retraction of consolidation, which might mean a better clinical course in this case. Based on the results shown in Table 2, most diseases that potentially show similar shadows in CT images were thought to be excluded.

Because ESKD patients are generally recognized as compromised hosts, they are inevitably supposed to be vulnerable to SARS-CoV-2 infection [15]. Moreover, this reported case was diabetic with heart disease requiring a pacemaker implantation. This case thus had higher risk for infection and progression of COVID-19. The patient fortunately recovered from critical condition by combination of possible management.

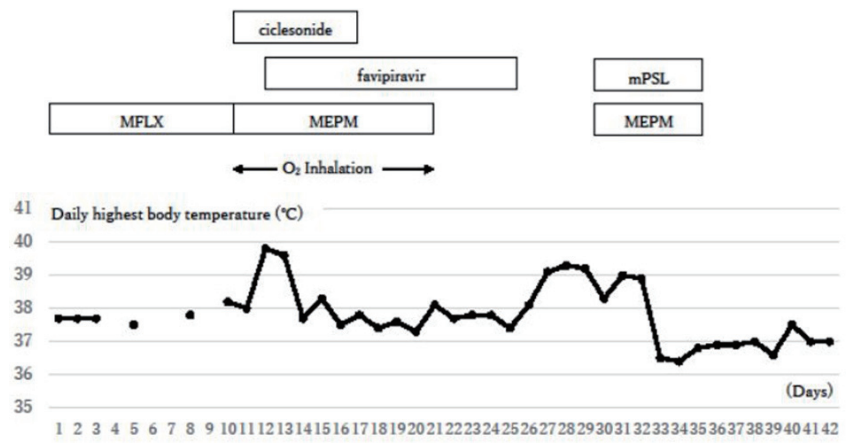

Figure 4. Clinical course. The inflammation signs were initially reduced with the treatments against COVID-19, but they flared up again around day 26. Additional steroid therapy against a possible cytokine storm succeeded in reducing symptoms. COVID-19: coronavirus disease 2019; $\mathrm{O}_{2}$ inhalation: oxygen inhalation of $1-2 \mathrm{~L} / \mathrm{min}$ through a nasal cannula; MFLX: moxifloxacin; MEPM: meropenem; mPSL: methyl prednisolone.
These experiences would be helpful to clarify and to establish a more appropriate protocol for treatment of COVID-19 in ESKD population.

Among drugs against COVID-19, we selected favipiravir and ciclesonide, based on their availability, effectiveness, and safety especially in ESKD of this case. For this treatment, we registered the case for ongoing clinical trials [16] and the informed consent was obtained from the patient. As described in Case Report, inflammation signs were reduced transiently, but then these signs flared up again. As reported previously [17], the cytokine storm was highly suspected due to an elevated IL-6 level on day 28. Because IL-6 is involved in developing the cytokine storm, and its circulating levels were correlated with disease severity in COVID-19 patients [17]. Moreover, serum levels of D-dimers were also elevated, which also suggested the cytokine storm [18]. We therefore administered methyl prednisolone along with MEPM to prevent secondary infection. Consequently, these treatments succeeded in suppression of inflammation to improve his general condition.

\section{Conclusions}

Since COVID-19 is emerging in December 2019 and has rapidly spread to all over the world, physician's experience in its diagnosis and treatment is still limited. RT-PCR methods are certainly the most reliable, but their limitations should be recognized by practitioners as reported here. We hope our experience in this case will give useful information to clinical staff confronting pandemic of COVID-19 around the world.

\section{Acknowledgments}

The authors appreciate all the staff engaged in the care of COVID-19 patients at JA Toride Medical Center.

\section{Financial Disclosure}

The authors have nothing to declare. 


\section{Conflict of Interest}

The authors state that they have no conflict of interest.

\section{Informed Consent}

The authors explained the significance of this report and obtained the written informed consent from the patient.

\section{Author Contributions}

The corresponding author, YM, mainly described the manuscript. Other authors were engaged in treatment of the patient, and carefully corrected the manuscript according to their experience.

\section{Data Availability}

The authors consider essential information is included in the manuscript. However, we will be ready to respond to requests for additional information on this report.

\section{References}

1. Jin Y, Yang H, Ji W, et al. Virology, epidemiology, pathogenesis, and control of COVID-19. Viruses. 2020;12:372.

2. IwasakiA, Grubaugh ND. Why does Japan have so few cases of COVID-19? EMBO Mol Med. 2020;12(5):e12481.

3. Karako K, Song P, Chen Y, Tang W. Analysis of COVID-19 infection spread in Japan based on stochastic transition model. Biosci Trends. 2020;14(2):134-138.

4. Xiao AT, Tong YX, Zhang S. False negative of RT-PCR and prolonged nucleic acid conversion in COVID-19: Rather than recurrence. J Med Virol. 2020.

5. National Institute of Infectious Diseases: Manual for the detection of pathogen 2019-nCoV. Ver. 2.6. February 17, 2020.

6. Wang W, Xu Y, Gao R, Lu R, Han K, Wu G, Tan W. Detection of SARS-CoV-2 in different types of clinical specimens. JAMA. 2020.
7. Kucirka LM, Lauer SA, Laeyendecker O, Boon D, Lessler $\mathrm{J}$. Variation in false-negative rate of reverse transcriptase polymerase chain reaction-based SARS-CoV-2 tests by time since exposure. Ann Intern Med. 2020;173(4):262267.

8. Osorio NS, Correia-Neves M. Implication of SARSCoV-2 evolution in the sensitivity of RT-qPCR diagnostic assays. Lancet Infect Dis. 2020.

9. Ai T, Yang Z, Hou H, Zhan C, Chen C, Lv W, Tao Q, et al. Correlation of chest $\mathrm{CT}$ and RT-PCR testing for coronavirus disease 2019 (COVID-19) in China: a report of 1014 cases. Radiology. 2020;296(2):E32-E40.

10. Lassauniere R, Frische A, Harboe ZB, et al. Evaluation of nine commercial SARS-Cov-2 immunoassays. medRxiv. 2020.

11. Food and Drug Administration (FDA, the U.S.A.): EUA authorized serology test performance. June 9, 2020. https://www.fda.gov/medical-devices/emergency-situations-medical-devices/eua-authorized-serology-test-performance.

12. $\mathrm{Li} \mathrm{J}, \mathrm{Xu}$ G. Lessons from the experience in Wuhan to reduce risk of COVID-19 infection in patients undergoing long-term hemodialysis. Clin J Am Soc Nephrol. 2020;15(5):717-719.

13. Bernheim A, Mei X, Huang M, et al. Chest CT findings in Coronavirus disease 2019 (COVID-19): relationship to duration of infection. Radiol. 2020;295:685-691.

14. Jajodia A, Ebner L, Heidinger B, K CA, Prosch H. Imaging in corona virus disease 2019 (COVID-19)-A scoping review. Eur J Radiol Open. 2020:100237.

15. Valeri AM, Robbins-Juarez SY, Stevens JS, Ahn W, Rao MK, Radhakrishnan J, Gharavi AG, et al. Presentation and outcomes of patients with ESKD and COVID-19. J Am Soc Nephrol. 2020;31(7):1409-1415.

16. Ito K, Ohmagari N, Mikami A, Sugiura W. Major ongoing clinical trials for COVID-19 treatment and studies currently being conducted or scheduled in Japan. Glob Health Med. 2020;2:96-101.

17. Coperchini F, Chiovato L, Croce L, Magri F, Rotondi M. The cytokine storm in COVID-19: An overview of the involvement of the chemokine/chemokine-receptor system. Cytokine Growth Factor Rev. 2020;53:25-32.

18. Jose RJ, Manuel A. COVID-19 cytokine storm: the interplay between inflammation and coagulation. Lancet Respir Med. 2020;8(6):e46-e47. 